\title{
Milnor and Tjurina numbers for smoothings of surface singularities
}

\author{
Jonathan Wahl \\ To Eduard Looijenga on his 65th birthday
}

\begin{abstract}
For an isolated hypersurface singularity $\{f=0\}$, the Milnor number $\mu$ is greater than or equal to the Tjurina number $\tau$ (the dimension of the base of the semi-universal deformation), with equality if $f$ is quasi-homogeneous. Saito proved the converse. The same result is true for complete intersections, but is much harder. For a Gorenstein surface singularity $(V, 0)$, the difference $\mu-\tau$ can be defined whether or not $(V, 0)$ is smoothable; the author has proved that it is non-negative, and equal to 0 if and only if $(V, 0)$ is quasi-homogeneous. We conjecture a similar result for non-Gorenstein surface singularities. Here, $\mu-\tau$ must be modified so that it is independent of any smoothing. This expression, involving cohomology of exterior powers of the bundle of logarithmic derivations on the minimal good resolution, is conjecturally non-negative, with equality if and only if $(V, 0)$ is quasi-homogeneous. We prove the "if" part; identify special cases where the conjecture is particularly interesting; verify it in some non-trivial cases; and prove it for a $\mathbb{Q}$-Gorenstein smoothing when the index one cover is a hypersurface. This conjecture arose regarding the classification of surface singularities with rational homology disk smoothings.
\end{abstract}

\section{Introduction}

Suppose that the $n$-dimensional hypersurface $V=\left\{f\left(z_{1}, \ldots, z_{n+1}\right)=0\right\} \subset \mathbb{C}^{n+1}$ has an isolated singularity at the origin. Then the Milnor fibre $M$ of $(V, 0)$ has the homotopy type of a bouquet of a certain number $\mu$ of $n$-spheres, where the Milnor number $\mu$ is the length of the Jacobian algebra

$$
J_{f}=\mathbb{C}\left[\left[z_{1}, \ldots, z_{n+1}\right]\right] /\left(\partial f / \partial z_{1}, \ldots, \partial f / \partial z_{n+1}\right) .
$$

The base space of the semi-universal deformation of $(V, 0)$ has dimension $\tau$, which is the length of the Tjurina algebra

$$
T_{f}=\mathbb{C}\left[\left[z_{1}, \ldots, z_{n+1}\right]\right] /\left(f, \partial f / \partial z_{1}, \ldots, \partial f / \partial z_{n+1}\right) .
$$

Received 11 September 2013, accepted in final form 7 November 2014.

2010 Mathematics Subject Classification 14J17, 14B07, 32S30, 32S25

Keywords: surface singularity, Milnor number, Tjurina number, smoothing, quasi-homogeneity, $\mathbb{Q}$-Gorenstein smoothing

This journal is (C) Foundation Compositio Mathematica 2015. This article is distributed with Open Access under the terms of the Creative Commons Attribution Non-Commercial License, which permits non-commercial reuse, distribution, and reproduction in any medium, provided that the original work is properly cited. For commercial re-use, please contact the Foundation Compositio Mathematica. 


\section{J. WAHL}

Clearly, $\mu \geqslant \tau$, with equality if and only if $f$ belongs to the Jacobian ideal. This is the case when $f$ is a weighted homogeneous polynomial. Saito [Sai71] proved the converse: equality implies that $f$ is quasi-homogeneous, that is, analytically equivalent to such a polynomial.

A local complete intersection singularity $(V, 0)$ of dimension $n \geqslant 1$ also has a Milnor fibre $M$ which is a bouquet of a certain number $\mu$ of $n$-spheres. The base space of its semi-universal deformation is again smooth of dimension $\tau=l\left(\operatorname{Ext}_{R}^{1}\left(\Omega_{R}^{1}, R\right)\right)$, where $l$ denotes length and $R$ is the local ring $\mathcal{O}_{V, 0}$. In this case it is harder to relate the size of the two invariants, which are dimensions of very different-looking spaces. But it was eventually proved by work of (among others) Greuel [Gre80], Looijenga-Steenbrink [LS85], the author [Wah85], and Vosegaard [Vos02] that also in these cases one has $\mu \geqslant \tau$, with equality if and only if $(V, 0)$ is quasi-homogeneous.

Smoothings for $(V, 0)$ a general normal surface singularity were investigated in [Wah81]. A singularity (for example, the cone over the rational quartic curve in $\mathbb{P}^{4}$ ) can have several topologically distinct smoothings, occurring over "smoothing components" of different dimension in the base space of the semi-universal deformation of $(V, 0)$. Thus, for a smoothing $\pi:(\mathcal{V}, 0) \rightarrow$ $(\mathbb{C}, 0)$, a Milnor fibre $M$ may be defined, but the rank $\mu_{\pi}$ of $H^{2}(M)$ and the dimension $\tau_{\pi}$ of the corresponding smoothing component depend on the smoothing. (It is known that the first Betti number of the Milnor fibre is zero, by [GS83]). Define $\alpha_{\pi}$ to be the colength of the restriction map of the dual of (relative) dualizing sheaves

$$
\omega_{\mathcal{V} / \mathbb{C}}^{*} \otimes \mathcal{O}_{V} \rightarrow \omega_{V}^{*}
$$

The following theorem was proved in special cases in [Wah81], and in general modulo several conjectures which were later established by Greuel, Looijenga, and Steenbrink ([GS83, GL85, Loo86]).

THEOREM $1.1([$ Wah81]). Let $\pi:(\mathcal{V}, 0) \rightarrow(\mathbb{C}, 0)$ be a smoothing of a normal surface singularity $(V, 0)$, with $\mu_{\pi}, \tau_{\pi}, \alpha_{\pi}$ as above. Let $(X, E) \rightarrow(V, 0)$ be a good resolution. Then

(i) $1+\mu_{\pi}=\alpha_{\pi}+13 h^{1}\left(\mathcal{O}_{X}\right)+\chi_{T}(E)-h^{1}\left(-K_{X}\right)$;

(ii) $\tau_{\pi}=2 \alpha_{\pi}+12 h^{1}\left(\mathcal{O}_{X}\right)+h^{1}\left(\Theta_{X}\right)-2 h^{1}\left(-K_{X}\right)$.

If $(V, 0)$ is Gorenstein, then $\alpha_{\pi}=0$, so $\mu$ and $\tau$ are independent of the smoothing.

In the Gorenstein case, one may use the expressions from the preceding theorem to define (possibly negative) singularity invariants $\mu$ and $\tau$, independent of smoothability. With this definition, we have in general the following result.

Theorem 1.2 ([Wah85]). If $(V, 0)$ is a Gorenstein surface singularity, then $\mu-\tau \geqslant 0$, with equality if and only if $(V, 0)$ is weighted homogeneous.

The point of this work is to study non-Gorenstein (for example, rational) surface singularities. Rather than $\mu-\tau$, consider the intrinsic invariant

$$
\left(\mu_{\pi}-\tau_{\pi}\right)+\alpha_{\pi},
$$

depending only on $(V, 0)$. A more useful version of Theorem 1.1 is the following, given in Section 2

Corollary 2.5. Let $(X, E) \rightarrow(V, 0)$ be the minimal good resolution of a normal surface singularity, not a rational double point. Denote by $S_{X}=\left(\Omega_{X}^{1}(\log (E))^{*}\right.$ the sheaf of derivations on $X$, logarithmic along $E$. Then for any smoothing $\pi$, one has

$$
1+\left(\mu_{\pi}-\tau_{\pi}\right)+\alpha_{\pi}=h^{1}\left(\mathcal{O}_{X}\right)-h^{1}\left(S_{X}\right)+h^{1}\left(\wedge^{2} S_{X}\right)
$$




\section{Milnor AND TJURINA NUMBERS FOR SMOOTHINGS OF SURFACE SINGULARITIES}

In the Gorenstein case, $\alpha_{\pi}=0$ and the methods of [Wah85] allow one to compute the right-hand side, particularly the difficult term $h^{1}\left(S_{X}\right)$ (whose dimension can vary in an "equisingular family"). Note that $h^{1}\left(\wedge^{2} S_{X}\right)=h^{1}\left(-\left(K_{X}+E\right)\right)$ is the second plurigenus $\delta_{2}(V)$ of $\mathrm{K}$. Watanabe ([Wat80]; see Lemma 2.1 below). It is not so easy to compute even for rational or quasi-homogeneous singularities.

The main purpose of this paper is to offer the following conjecture.

Conjecture (Main Conjecture). Let $(X, E) \rightarrow(V, 0)$ be the minimal good resolution of a nonGorenstein normal surface singularity. Denoting by $S_{X}$ the sheaf of logarithmic derivations on $X$, one has

$$
h^{1}\left(\mathcal{O}_{X}\right)-h^{1}\left(S_{X}\right)+h^{1}\left(\wedge^{2} S_{X}\right) \geqslant 0,
$$

with equality if and only if $(V, 0)$ is quasi-homogeneous.

The relevant expression $\sum_{i=0}^{2}(-1)^{i} h^{1}\left(\wedge^{i} S_{X}\right)$ looks somewhat like a second Chern class, as explained in Proposition 2.7.

The Main Conjecture (given also in Conjecture 2.6 below) might be overly optimistic. One can check it in certain cases by computing the right or left side in Corollary 2.5.

One implication is proved in this paper.

TheOrem 3.3. If $(V, 0)$ is quasi-homogeneous and not Gorenstein, then

$$
h^{1}\left(\mathcal{O}_{X}\right)-h^{1}\left(S_{X}\right)+h^{1}\left(\wedge^{2} S_{X}\right)=0 .
$$

Certain special cases are worth pursuing. In Section 4, we have the following conjecture.

Conjecture 4.1 (Rational Conjecture). Let $(X, E) \rightarrow(V, 0)$ be the minimal good resolution of a rational surface singularity, not an RDP (rational double point). Then

$$
h^{1}\left(S_{X}\right) \leqslant h^{1}\left(-\left(K_{X}+E\right)\right),
$$

with equality if and only if $(V, 0)$ is quasi-homogeneous.

Recall that $h^{1}\left(S_{X}\right)$ is the dimension of the smooth space of equisingular deformations of $(V, 0)$, obtained from the deformations of $X$ which preserve every exceptional curve [Wah76]. As for $h^{1}\left(-\left(K_{X}+E\right)\right)$, we prove in Corollary 4.5 that it can be computed from the resolution graph; thus the conjecture would give a topological upper bound for the dimension $h^{1}\left(\Theta_{X}\right)$ of the Artin component of $(V, 0)$. As evidence for the Rational Conjecture, we show in Section 4 validity in several cases:

(i) rational singularities for which $h^{1}\left(S_{X}\right)=0$ (Proposition 4.9);

(ii) rational singularities for which the resolution graph of $(V, 0)$ is star-shaped (Proposition 4.8);

(iii) an example with non-star-shaped graph and $h^{1}\left(S_{X}\right)=1$ (Example 4.10).

In a forthcoming paper, we verify the conjecture (in a stronger form) for any rational graph which is "sufficiently negative at the nodes"; see Remark 4.11 for a precise statement.

Recall that $(V, 0)$ is called $\mathbb{Q}$-Gorenstein if $K_{V}$ is $\mathbb{Q}$-Cartier, that is, some $r K_{V}$ is invertible; then $(V, 0)$ is an $r$-cyclic quotient of its index one (or canonical) cover $(W, 0)$, which is Gorenstein. A smoothing $\pi:(\mathcal{V}, 0) \rightarrow(\mathbb{C}, 0)$ is called $\mathbb{Q}$-Gorenstein if it is an $r$-cyclic quotient of a smoothing of $(W, 0)$. In this case, $\alpha_{\pi}=0$ (see Lemma 5.1), so the conjecture is again about $\mu-\tau$. We discuss in Section 5 the following conjecture. 


\section{J. WAHL}

Conjecture $(\mathbb{Q}$-Gorenstein Conjecture). For a $\mathbb{Q}$-Gorenstein smoothing of a non-Gorenstein singularity $(V, 0)$, one has

$$
\mu \geqslant \tau-1,
$$

with equality if and only if $(V, 0)$ is quasi-homogeneous.

We verify this last conjecture in the case that the canonical cover is a hypersurface singularity. In fact, a much more general result, in all dimensions, is proved.

Theorem 5.2. Let $(W, 0)$ be an isolated hypersurface singularity $\left(\left\{f\left(z_{1}, \cdots, z_{n+1}\right)=0\right\}, 0\right) \subset$ $\left(\mathbb{C}^{n+1}, 0\right)$, and let $G \subset \mathrm{GL}(n+1, \mathbb{C})$ be a finite group acting freely off 0 and leaving $f$ invariant. The map $f:\left(\mathbb{C}^{n+1} / G, 0\right) \rightarrow(\mathbb{C}, 0)$ is a $\mathbb{Q}$-Gorenstein smoothing of $(V, 0) \equiv(W / G, 0)$, with smoothing invariants $\bar{\mu}$ and $\bar{\tau}$.

(i) If $G \subset \mathrm{SL}(n+1, \mathbb{C})$, then $(V, 0)$ is Gorenstein and $\bar{\mu} \geqslant \bar{\tau}$, with equality if and only if $(V, 0)$ is quasi-homogeneous.

(ii) If $G \not \subset \mathrm{SL}(n+1, \mathbb{C})$, then $(V, 0)$ is not Gorenstein and $\bar{\mu} \geqslant \bar{\tau}-1$, with equality if and only if $(V, 0)$ is quasi-homogeneous.

(The Milnor fibre for the quotient smoothing again has rational homology only in dimension $n$, of rank $\bar{\mu}$ ). The key ingredients of the proof are the main results of [Wal80] and [GL85] plus the Lefschetz fixed-point theorem.

A normal surface singularity in $\left(\mathbb{C}^{4}, 0\right)$ is smoothable, with a smooth base space for the semiuniversal deformation. Gorenstein examples are complete intersections. It is proved in [Wah81, (3.14.4)] that $\alpha$ is zero. The conjecture in Section 6 is as follows.

Conjecture 6.1 (Codimension Two Conjecture). Let $(V, 0)$ be a normal surface singularity in $\left(\mathbb{C}^{4}, 0\right)$, not a complete intersection. Then

$$
\mu \geqslant \tau-1,
$$

with equality if and only if $(V, 0)$ is quasi-homogeneous.

Examples of $(V, 0) \subset\left(\mathbb{C}^{4}, 0\right)$ which are neither $\mathbb{Q}$-Gorenstein nor quasi-homogeneous, with $\mu=\tau$ (consistently with the conjecture), can be found in Example 6.2.

Our original motivation for the conjecture (even for rational singularities) concerns those $(V, 0)$ admitting a "rational homology disk smoothing," that is, a smoothing with Milnor number zero. These are especially interesting to topologists because the link of such a singularity possesses a symplectic filling with no rational homology. A complete classification exists in case $(V, 0)$ is quasi-homogeneous ([SSW08, BS11]), and it was conjectured in [Wah11] that these are the only examples. If a $(\mu=0)$-smoothing were $\mathbb{Q}$-Gorenstein (as happens in the quasi-homogeneous case [Wah13]), one would have $\alpha=0$ and $\tau \geqslant 1$, whence (assuming the Rational or $\mathbb{Q}$-Gorenstein Conjecture) $\tau=1$ and $(V, 0)$ would be quasi-homogeneous. Thus, there would be no other examples with $\mathbb{Q}$-Gorenstein smoothings.

Note finally that there exist similar results and conjectures for a reduced curve singularity $(C, 0)$. Here, $\mu$ is defined by Buchweitz-Greuel [BG80], and $\tau$ (the dimension of a smoothing component) is (via Deligne [Del73]) an expression in terms of curve invariants. Work of Greuel and others ([Gre81, GMP85]) gives the following results:

(i) For $(C, 0)$ Gorenstein, one has $\mu \geqslant \tau$, with equality if and only if $(C, 0)$ is quasi-homogeneous. 


\section{Milnor AND TJURINA NUMBERS FOR SMOOTHINGS OF SURFACE SINGULARITIES}

(ii) For $(C, 0)$ quasi-homogeneous, one has $\mu=\tau+1-t$, where $t$ (the "type" of $(C, 0))$ is the minimal number of generators of the dualizing sheaf $\omega_{C}$.

(iii) (Conjecture) One has $\mu \geqslant \tau+1-t$, with equality if and only if $(C, 0)$ is quasi-homogeneous.

\section{Formulas for $\mu$ and $\tau$}

Formulas for $\mu$ and $\tau$ for a smoothing $\pi:(\mathcal{V}, 0) \rightarrow(\mathbb{C}, 0)$ are given in Theorem 3.13 of [Wah81], modulo three conjectures, proved there only in certain cases, but later established in general. Specifically, the first Betti number of the Milnor fibre is zero ([GS83]); any smoothing can be appropriately globalized ([Loo86]); and the dimension of the smoothing component corresponding to $\pi$ is the length of the cokernel of

$$
\Theta_{\mathcal{V} / \mathbb{C}} \otimes \mathcal{O}_{V} \rightarrow \Theta_{V}
$$

(see [GL85]). The invariant measuring the change in the dual of the dualizing sheaves is

$$
\alpha=l\left(\operatorname{Coker}\left(\omega_{\mathcal{V} / \mathbb{C}}^{*} \otimes \mathcal{O}_{V} \rightarrow \omega_{V}^{*}\right)\right) .
$$

Writing $R=\mathcal{O}_{(V, 0)}$ for the local ring of $V$ at 0 , one has ([Wah81, Corollary A.2])

$$
0 \leqslant \alpha \leqslant l\left(\operatorname{Ext}_{R}^{1}(\omega, R)\right) .
$$

One deduces that $\alpha=0$ if $(V, 0)$ is Gorenstein or a normal surface in $\mathbb{C}^{4}$.

We rewrite the formulas relating $\mu, \tau$, and $\alpha$ not in terms of $\Theta_{X}$ and its second exterior power $-K_{X}$, but rather in terms of the sheaf $S=S_{X}$ of derivations logarithmic along $E$, and its second exterior power $-\left(K_{X}+E\right)$.

Lemma 2.1 ([Oku97]). Let $(X, E) \rightarrow(V, 0)$ be the minimal good resolution of a normal surface singularity, not an RDP. Then

(i) $H_{E}^{1}\left(X,-\left(K_{X}+E\right)\right)=0$;

(ii) the dimension $h^{1}\left(X,-\left(K_{X}+E\right)\right)$ equals the second plurigenus

$$
\left.\delta_{2}(V)=\operatorname{dim} H^{0}\left(X-E, 2 K_{X}+E\right) / H^{0}\left(X, 2 K_{X}+E\right)\right) .
$$

Proof. Corollary 1.9 of [Oku97] asserts that $h^{1}\left(2 K_{X}+E\right)=0$, whence by the long exact sequence in local cohomology $\delta_{2}(V)=h_{E}^{1}\left(2 K_{X}+E\right)$. Local duality for a line bundle $L$ on $X$ yields $h_{E}^{1}(L)=h^{1}\left(K_{X}-L\right)$, from which both assertions follow.

Proposition 2.2. Let $\pi:(\mathcal{V}, 0) \rightarrow(\mathbb{C}, 0)$ be a smoothing of a normal surface singularity $(V, 0)$, not an RDP, with $\mu, \tau$, and $\alpha$ as before. Let $(X, E) \rightarrow(V, 0)$ be the minimal good resolution. Then

(i) $1+\mu=\alpha+13 h^{1}\left(\mathcal{O}_{X}\right)+\chi_{T}(E)-(1 / 2) E \cdot(E+3 K)-h^{1}\left(-\left(K_{X}+E\right)\right)$;

(ii) $\tau=2 \alpha+12 h^{1}\left(\mathcal{O}_{X}\right)+\chi_{T}(E)-(1 / 2) E \cdot(E+3 K)+h^{1}(S)-2 h^{1}\left(-\left(K_{X}+E\right)\right)$.

Proof. For each exceptional curve $E_{i}$, denote the genus by $g_{i}$, the degree by $-d_{i}$, and the number of intersections with other curves by $t_{i}$. One has the standard short exact sequence

$$
0 \rightarrow S \rightarrow \Theta \rightarrow \oplus N_{E_{i}} \rightarrow 0,
$$

where $N_{E_{i}}$ is the normal bundle of $E_{i}$. Since $h^{0}\left(N_{E_{i}}\right)=0$, one has $h^{1}\left(N_{E_{i}}\right)=g_{i}+d_{i}-1$, so

$$
h^{1}(\Theta)=h^{1}(S)+\sum\left(g_{i}+d_{i}-1\right) .
$$




\section{J. WAHL}

Lemma 2.3. Let $(X, E) \rightarrow(V, 0)$ be the minimal good resolution of a normal surface singularity, not an RDP. Then

$$
H^{0}\left(X,\left(-K_{X}\right) \otimes \mathcal{O}_{E}\right)=0 .
$$

Proof. The group $H_{E}^{1}\left(-\left(K_{X}+E\right)\right)$ is the direct limit of the direct system given by the injective maps

$$
H^{0}\left(-\left(K_{X}+E\right) \otimes \mathcal{O}_{Z}(Z)\right) \rightarrow H^{0}\left(-\left(K_{X}+E\right) \otimes \mathcal{O}_{Z+Z^{\prime}}\left(Z+Z^{\prime}\right)\right),
$$

where $Z$ and $Z^{\prime}$ are effective exceptional divisors. As $h_{E}^{1}\left(-\left(K_{X}+E\right)\right)=0$, each of these spaces is 0 , in particular for $Z=E$, as desired.

Lemma 2.4. Let $(X, E) \rightarrow(V, 0)$ be the minimal good resolution of a normal surface singularity, not an RDP. Then

$$
h^{1}\left(X,-K_{X}\right)=h^{1}\left(X,-\left(K_{X}+E\right)\right)+(1 / 2) E \cdot(E+3 K) .
$$

Proof. By Lemma 2.3 and the standard exact sequence, it suffices to compute $h^{1}\left(\left(-K_{X}\right) \otimes \mathcal{O}_{E}\right)=$ $-\chi\left(\left(-K_{X}\right) \otimes \mathcal{O}_{E}\right)$. The Riemann-Roch theorem for a line bundle $L$ and any exceptional divisor $Z$ states

$$
\chi\left(L \otimes \mathcal{O}_{Z}\right)=(-1 / 2) Z \cdot(Z+K)+Z \cdot L,
$$

from which the result follows.

Proof of Proposition 2.2, continued. Lemma 2.4 provides the formula for $1+\mu$. The formula for $\tau$ requires checking the easily verified relation

$$
\chi_{T}(E)=\sum\left(g_{i}+d_{i}-1\right)-E \cdot(E+3 K) / 2 .
$$

COROLlary 2.5. With notation as before, for a smoothing of a normal surface singularity (not an $R D P$ ), one has

$$
1+(\mu-\tau)+\alpha=h^{1}\left(\mathcal{O}_{X}\right)-h^{1}\left(S_{X}\right)+h^{1}\left(-\left(K_{X}+E\right)\right) .
$$

This allows one to formulate the Main Conjecture of the paper, which is motivated by (but independent of) smoothing questions.

Conjecture 2.6. Let $(X, E) \rightarrow(V, 0)$ be the minimal good resolution of a normal surface singularity, not an RDP.

(i) If $(V, 0)$ is Gorenstein, then $h^{1}\left(\mathcal{O}_{X}\right)-h^{1}\left(S_{X}\right)+h^{1}\left(-\left(K_{X}+E\right)\right) \geqslant 1$, with equality if and only if $(V, 0)$ is quasi-homogeneous.

(ii) If $(V, 0)$ is not Gorenstein, then $h^{1}\left(\mathcal{O}_{X}\right)-h^{1}\left(S_{X}\right)+h^{1}\left(-\left(K_{X}+E\right)\right) \geqslant 0$, with equality if and only if $(V, 0)$ is quasi-homogeneous.

As previously mentioned, assertion (i) has been proved in [Wah85].

The relevant expression in the conjecture can be written in an alternative and suggestive way. The standard Euler characteristic of a locally free sheaf $\mathcal{F}$ on $X$ is

$$
\chi(\mathcal{F})=\operatorname{dim} H^{0}(X-E, \mathcal{F}) / H^{0}(X, \mathcal{F})-\operatorname{dim} H^{1}(\mathcal{F}) .
$$

If $H_{E}^{1}(\mathcal{F})=0$, one has simply $\chi(\mathcal{F})=-h^{1}(\mathcal{F})$. Now, in the case at hand, one has

(i) $h_{E}^{1}\left(\wedge^{0} S_{X}\right)=h_{E}^{1}\left(\mathcal{O}_{X}\right)=0$, by Grauert-Riemenschneider;

(ii) $h_{E}^{1}\left(S_{X}\right)=0$, by the main theorem of [Wah75]; 
Milnor And TJurina numbers For SMOOThings of SURface SINGUlarities

(iii) $h_{E}^{1}\left(\wedge^{2}\left(S_{X}\right)=0\right.$, by [Oku97], as in Lemma 2.1 .

Proposition 2.7. On the minimal good resolution $X$ of a normal surface singularity, one has

$$
h^{1}\left(\mathcal{O}_{X}\right)-h^{1}\left(S_{X}\right)+h^{1}\left(-\left(K_{X}+E\right)\right)=-\sum_{i=0}^{2}(-1)^{i} \chi\left(\wedge^{i} S_{X}\right) .
$$

It is interesting to compare with the Riemann-Roch theorem for a rank two vector bundle $\mathcal{F}$ on a smooth projective surface $Y$, which yields

$$
\sum_{i=0}^{2}(-1)^{i} \chi\left(Y, \wedge^{i} \mathcal{F}\right)=c_{2}(\mathcal{F}) .
$$

\section{The quasi-homogeneous case}

The following result was asserted in [Wah81, (4.10.2)], but not carefully proved there.

Proposition 3.1. Let $(X, E) \rightarrow(V, 0)$ be the minimal good resolution of a quasi-homogeneous surface singularity, not a cyclic quotient. Then the Euler derivation $D$ of $(V, 0)$ induces a nowherezero section of the vector bundle $S_{X}$, hence gives a short exact sequence

$$
0 \rightarrow \mathcal{O}_{X} \rightarrow S_{X} \rightarrow \wedge^{2}\left(S_{X}\right) \rightarrow 0 .
$$

We may assume that $V=\operatorname{Spec} A$ is an affine variety, where $A=\oplus A_{i}$ is a positively graded normal domain. Write $A=\mathbb{C}\left[z_{1}, \ldots, z_{s}\right] /\left(g_{\alpha}\left(z_{i}\right)\right)$, a quotient of a graded polynomial ring, where $\operatorname{deg} z_{i}=m_{i}$. The Euler derivation on $A_{i}$ is multiplication by $m_{i}$, and $D=\sum_{i=1}^{s} m_{i} z_{i} \partial / \partial z_{i}$.

Let $W \rightarrow V$ be the partial resolution obtained by blowing up the weight filtration of $A$. The variety $W$ has cyclic quotient singularities along the (smooth) exceptional curve $C$, which is isomorphic to Proj $A$. Minimally resolving these cyclic quotients gives $X$ and $E$, which provides the minimal good resolution. The derivation $D$ lifts to a section of both $\Theta_{W}$ and $\Theta_{X}$; as $H^{0}\left(S_{X}\right)=$ $H^{0}\left(\Theta_{X}\right)$, it is also a section of $S_{X}$. We must show that it is nowhere zero. This is clear on $X-E=V-\{0\}$.

The variety $W$ is the union of $s$ affines $W_{i}$, each the quotient of a smooth affine $U_{i}$ by a cyclic group of order $m_{i}$. To define $U_{1}$, write

$$
z_{1}=x^{m_{1}}, z_{2}=x^{m_{2}} y_{2}, \ldots, z_{s}=x^{m_{s}} y_{s} .
$$

Then $U_{1}$ is the affine variety with coordinate ring

$$
\mathbb{C}\left[x, y_{2}, \ldots, y_{s}\right] /\left(g_{\alpha}\left(1, y_{2}, \ldots, y_{s}\right)\right),
$$

hence is polynomial in $x$. The derivation $D$ lifts to the derivation $x \partial / \partial x$, and is a nowhere-zero section of the bundle of derivations on $U_{1}$ logarithmic along $x=0$. The variety $W_{1}$ is the quotient of $U_{1}$ by the cyclic group of order $m_{1}$ generated by

$$
T=\left(1 / m_{1}\right)\left[-1, m_{2}, \ldots, m_{s}\right] .
$$

If the action is free at a point of $x=0$, the quotient map is a local analytic isomorphism; so at the corresponding point of $C \subset W_{1}$, the derivation $D$ is still a nowhere-zero section of the corresponding bundle of logarithmic derivations. At points of $x=0$ where there is isotropy (that is, above the cyclic quotient singularities of $W_{1}$ ), consider the corresponding local analytic model on $U_{1}$. 


\section{J. WAHL}

Changing notation slightly, for local analytic coordinates $x, y$ on $\mathbb{C}^{2}$, consider the action of $\mathbb{Z} / r$ of type $1 / r[1, a]$ and the cyclic quotient singularity $W=\mathbb{C}^{2} /(\mathbb{Z} / r)$. Let $X \rightarrow W$ be the minimal equivariant resolution of $W$, with exceptional divisor $E=\Sigma_{i=1}^{l} E_{i}$. Denote by $C$ the Weil divisor on $W$ given by the image of $x=0$, and by $C^{\prime}$ its proper transform on $Y$. Then $C^{\prime}$ intersects $E$ transversally along one end, say $E_{l}$. The following lemma will complete the proof of the proposition.

Lemma 3.2. The derivation $D=x \partial / \partial x$ on $\mathbb{C}^{2}$ induces a derivation of $W$ and lifts to a nowherezero section of the rank two vector bundle $\Omega_{X}^{1}\left(\log \left(E+C^{\prime}\right)\right)^{*}$ on $X$.

Proof. Use the familiar description (cf. Reid's Warwick notes [Rei, page 10]) of the minimal resolution as a union $X_{0} \cup X_{1} \cup \cdots \cup X_{l}$ of copies of $\mathbb{C}^{2}$. A copy $X_{i}$ has coordinates $u_{i}, v_{i}$, with the exceptional curve given by $v_{0}=0$ on $X_{0} ; u_{l}=0$ on $X_{l}$; and $u_{i} v_{i}=0$ on the intermediate $X_{i}$. The curve $C^{\prime}$ is given by $u_{0}=0$ on $X_{0}$. On $X_{i}$, we have $u_{i}=x^{a_{i}} y^{b_{i}}, v_{i}=x^{c_{i}} y^{d_{i}}$, for appropriate integer exponents. Thus on this affine, one has $D=a_{i} u_{i} \partial / \partial u_{i}+c_{i} v_{i} \partial / \partial v_{i}$. The construction shows that all the $a_{i}$ and $c_{i}$ are non-zero, except that $c_{l}=0$. Thus, $D$ has the desired property on every $X_{i}$.

Theorem 3.3. Let $(X, E) \rightarrow(V, 0)$ be the minimal good resolution of a quasi-homogeneous singularity, not an RDP.

(i) If $(V, 0)$ is not Gorenstein, then

$$
h^{1}\left(\mathcal{O}_{X}\right)-h^{1}(S)+h^{1}\left(-\left(K_{X}+E\right)\right)=0 .
$$

(ii) If $(V, 0)$ is Gorenstein, then

$$
h^{1}\left(\mathcal{O}_{X}\right)-h^{1}(S)+h^{1}\left(-\left(K_{X}+E\right)\right)=1 .
$$

Proof. As previously noted, the theorem has already been proved for Gorenstein singularities. It is also true for non-RDP cyclic quotients (since each individual term in (i) vanishes, for instance using Corollary 4.5 below).

For $(V, 0)$ non-Gorenstein, by Proposition 3.1 one must show that the (graded) coboundary map

$$
\delta: H^{0}\left(X,-\left(K_{X}+E\right)\right) \rightarrow H^{1}\left(X, \mathcal{O}_{X}\right)
$$

is zero. We examine the graded pieces of the map, using the notation and results of DemazureWatanabe in [Wat81]. The analytic data of $(V, 0)$ is given by the central curve $C=$ Proj $A$, its normal divisor $D$ on $X$, and cyclic quotient singularities of type $n_{i} / q_{i}$ at points $P_{i}$ for $i=1, \ldots, t$. Consider the $\mathbb{Q}$-divisors

$$
F=D-\sum\left(q_{i} / n_{i}\right) P_{i}, \quad \Xi=K+\sum\left(1-1 / n_{i}\right) P_{i} .
$$

Recall that for these types of $\mathbb{Q}$-divisors $G$, one defines an invertible sheaf

$$
\mathcal{O}(G) \equiv \mathcal{O}(\lfloor G\rfloor)) \subset k(C) .
$$

Pinkham's basic result is

$$
A=\oplus A_{k}=H^{0}\left(X, \mathcal{O}_{X}\right)=\oplus_{k=-\infty}^{\infty} H^{0}(C, k F) T^{k} .
$$

Watanabe proved (see [Wat81])

$$
\omega_{A}=H^{0}\left(X-E, K_{X}\right)=\oplus_{k=-\infty}^{\infty} H^{0}(C, \Xi+k F) T^{k} .
$$




\section{Milnor AND TJuRina NUMBERs FOR SMOOTHINGS OF SURFACE SINGUlARITIES}

It follows from the general constructions that

$$
\begin{aligned}
& H^{1}\left(X, \mathcal{O}_{X}\right)=\oplus_{k=0}^{\infty} H^{1}(C, k F) T^{k}, \\
& \omega_{A}^{*}=H^{0}\left(X-E,-K_{X}\right)=\oplus_{k=-\infty}^{\infty} H^{0}(C,-\Xi+k F) T^{k} .
\end{aligned}
$$

Since $H^{0}\left(X,-\left(K_{X}+E\right)\right) \subset H^{0}\left(X-E,-\left(K_{X}+E\right)\right)=\omega_{A}^{*}$, it follows that the $k$ th graded piece of the coboundary map factors through

$$
H^{0}(C,-\Xi+k F) \rightarrow H^{1}(C, k F) .
$$

The second space is dual to $H^{0}(C, K-\lfloor k F\rfloor)$; in order for the map to be non-zero, both this space and $H^{0}(C,\lfloor-\Xi+k F\rfloor)$ must be non-zero. Therefore, the sum of the corresponding integral divisors is effective. One checks that the sum is supported at the points $P_{i}$, with coefficients

$$
\left\lfloor-\left(\left(k q_{i}-1\right) / n_{i}+1\right)\right\rfloor-\left\lfloor-k q_{i} / n_{i}\right\rfloor .
$$

But this expression equals -1 , unless $k q_{i} \equiv 1 \bmod n_{i}$, in which case it is zero. Thus, a non-zero coboundary map requires $k q_{i} \equiv 1 \bmod n_{i}$ for all $i$. Since the sum of the two divisors is effective and of degree zero, and each divisor has a section, the divisors themselves would have to be trivial. This implies, in particular, $K=\lfloor k F\rfloor$. By Watanabe's criterion, $(V, 0)$ is Gorenstein.

It is not necessary to prove here the delicate fact that the coboundary map has rank one in the Gorenstein case [Hoc77].

\section{Rational surface singularities}

Conjecture 4.1 (Rational Conjecture). Let $(X, E) \rightarrow(V, 0)$ be the minimal resolution of a rational surface singularity, not an RDP. Then

$$
h^{1}\left(S_{X}\right) \leqslant h^{1}\left(-\left(K_{X}+E\right)\right),
$$

with equality if and only if $(V, 0)$ is quasi-homogeneous.

The dimension $h^{1}\left(S_{X}\right)$ of the space of equisingular deformations is very difficult to compute (but see Example 4.10 below). The term $h^{1}\left(-K_{X}\right)$ has been encountered in [Wah80, (1.12)], where it is proved that for $R$ the local ring of $(V, 0)$,

(i) $h^{1}\left(-K_{X}\right)=l\left(\operatorname{Ext}_{R}^{1}(\omega, R)\right)$;

(ii) $h^{1}\left(-K_{X}\right) \geqslant \operatorname{mult}(R)-3$.

As $E \cdot(E+K)=-2$, Lemma 2.3 implies

$$
h^{1}\left(-K_{X}\right)=h^{1}\left(-\left(K_{X}+E\right)\right)+((-E \cdot E)-3) .
$$

If $E$ is the fundamental cycle, $\operatorname{mult}(R)=-E \cdot E$, and $h^{1}\left(-\left(K_{X}+E\right)\right)$ calculates the difference in the second inequality above.

We show that $h^{1}\left(-\left(K_{X}+E\right)\right)$ can be calculated from the graph of $(V, 0)$. Recall the notation and results of Giraud [Gir82]. For a line bundle $L$ on a good resolution $(X, 0) \rightarrow(V, 0)$ of a normal surface singularity, define $[L]$ to be the smallest (in the usual ordering) integral divisor $D$ such that

$$
D \cdot E_{i} \leqslant L \cdot E_{i} \text { for all } i .
$$

Suppose that $L$ is numerically equivalent to $\sum a_{i} E_{i}$ with $a_{i} \in \mathbb{Q}$. One forms $[L]$ by first rounding up all $a_{i}$ to form an integral $D_{1}$, and inductively defining $D_{n+1}=D_{n}+E_{j}$ if one finds that $D_{n} \cdot E_{j}>L \cdot E_{j}$. 


\section{J. WAHL}

Theorem 4.2 (Giraud [Gir82]). Let $L$ be a line bundle on $X$ such that

$$
[L] \cdot E_{i} \leqslant 0 \quad \text { for all } E_{i} .
$$

Then one has $H_{E}^{1}(L)=0$.

For $L$ a line bundle and $D$ an integral divisor, one has $[L-D]=[L]-D$; in particular

$$
[(L-[L])]=0 .
$$

Corollary 4.3. For any line bundle $L$ on $X$, one has $H_{E}^{1}(L-[L])=0$.

Note that $[L]$ is an effective divisor if $L \cdot E_{i} \leqslant 0$ for all $i$, or more generally if the coefficients $a_{i}$ as above are all greater than -1 .

Proposition 4.4. On the minimal resolution $X$ of a rational singularity, let $L$ be a line bundle for which $[L] \equiv Z$ is an effective divisor. Then

$$
\operatorname{dim} H^{1}(X, L)=Z \cdot(Z+K) / 2-Z \cdot L .
$$

Proof. The result is easy if $Z=0$, so assume $Z>0$. By duality and Corollary 5.2, one has

$$
0=h_{E}^{1}(\mathcal{O}(L-Z))=h^{1}(\mathcal{O}(K-L+Z)),
$$

so that

$$
0=h^{1}\left(\mathcal{O}(K-L+Z) \otimes \mathcal{O}_{Z}\right)=h_{E}^{1}\left(\mathcal{O}(K-L+Z) \otimes \mathcal{O}_{Z}\right) .
$$

Consider the short exact sequence of sheaves on $X$,

$$
0 \rightarrow \mathcal{O}(K-L) \rightarrow \mathcal{O}(K-L+Z) \rightarrow \mathcal{O}(K-L+Z) \otimes \mathcal{O}_{Z} \rightarrow 0 .
$$

Taking local cohomology yields

$$
0 \rightarrow H_{E}^{0}\left(\mathcal{O}(K-L+Z) \otimes \mathcal{O}_{Z}\right) \rightarrow H_{E}^{1}(\mathcal{O}(K-L)) \rightarrow H_{E}^{1}(\mathcal{O}(K-L+Z)) \rightarrow 0 .
$$

The first term (because of the vanishing of the corresponding $H_{E}^{1}$ ) is the Euler characteristic of a line bundle on $Z$, so by the Riemann-Roch theorem its dimension is $Z \cdot(Z+K) / 2-Z \cdot L$. The middle term has dimension $h^{1}(L)$, and one must show $h_{E}^{1}(K-L+Z)=0$. As usual, it suffices to prove that for any effective cycle $Y$, there is an $E_{i}$ in the support of $Y$ such that

$$
(K-L+Z+Y) \cdot E_{i}<0 .
$$

By definition $(Z-L) \cdot E_{i} \leqslant 0$ for all $i$; so it suffices to find $(K+Y) \cdot E_{i}<0$. This is standard, because by rationality one always has $Y \cdot(Y+K) \leqslant-2$.

Corollary 4.5. Let $(X, E) \rightarrow(V, 0)$ be the minimal resolution of a rational surface singularity, not an RDP. Then

(i) for $Y=\left[-K_{X}\right]$, one has

$$
h^{1}\left(X,-K_{X}\right)=Y \cdot(Y+3 K) / 2 ;
$$

(ii) for $Z=\left[-\left(K_{X}+E\right)\right]=Y-E$, one has

$$
h^{1}\left(X,-\left(K_{X}+E\right)\right)=Z \cdot(Z+3 K) / 2+Z \cdot E ;
$$

(iii) in particular, these dimensions are topological and depend only on the graph of $E$.

Proof. Since $K \cdot E_{i} \geqslant 0$ for all $i$ and $K \neq 0$, one has $-K \equiv \sum a_{i} E_{i}$ with all $a_{i}>0$. Thus, both $[-K]$ and $[-(K+E)]$ are effective, so Proposition 4.4 applies. 


\section{Milnor AND TJuRina NUMBERs FOR SMOOTHINGS OF SURFACE SINGUlARITIES}

Remark 4.6. For a rational singularity, $[-K] \cdot E_{i} \leqslant 2-d_{i} \leqslant 0$ for all $i$, so that $[-K]$ is at least as big as the fundamental cycle $Z_{0}$. The easiest way to compute $[-K]$ (and hence $[-K]-E$ ) is to add curves to $Z_{0}$ until one reaches a cycle $Y$ satisfying $Y \cdot E_{i} \leqslant 2-d_{i}$ for all $i$.

Remark 4.7. It follows from [LW86, (4.11.4)] that if a normal surface singularity admits a $\mathbb{Q}$ Gorenstein smoothing, then $h^{1}\left(-K_{X}\right)=-K \cdot K+h^{1}\left(\mathcal{O}_{X}\right)$.

Proposition 4.8. Consider a rational surface singularity $(V, 0)$ with a star-shaped graph (not a cyclic quotient singularity), with $(X, E) \rightarrow(V, 0)$ the minimal resolution. Then the Main Conjecture holds for $(V, 0)$; that is,

$$
h^{1}\left(X, S_{X}\right) \leqslant h^{1}\left(X,-\left(K_{X}+E\right)\right),
$$

with equality if and only if $(V, 0)$ is quasi-homogeneous.

Proof. In the local ring of $V$, consider the filtration given by the order of vanishing along the central curve in the resolution. The associated graded ring $A$ for this filtration is normal, and is a quasi-homogeneous rational singularity with isomorphic resolution graph. The corresponding degeneration gives a deformation of $\operatorname{Spec} A$ whose general fibre is isomorphic to $(V, 0)$, and this family admits a simultaneous equisingular resolution (and, in fact, the reduced exceptional curve is analytically isomorphic). By Corollary 4.5, the dimension $h^{1}(-(K+E))$ is constant in the family, and it equals $h^{1}(S)$ on the special fibre. It suffices to show that if $h^{1}(S)$ is constant in the family, then the original singularity was already quasi-homogeneous. Let $C$ denote the central curve, and consider on any of the resolutions the exact sequence

$$
H^{0}(S) \rightarrow H^{0}\left(S \otimes \mathcal{O}_{C}\right) \rightarrow H^{1}(S(-C)) \rightarrow H^{1}(S) \rightarrow H^{1}\left(S \otimes \mathcal{O}_{C}\right) \rightarrow 0 .
$$

According to [Wah85, (3.11) and (3.2)], the second space has dimension one and the first map is surjective if and only if the singularity is quasi-homogeneous. Since the special fibre has

$$
h^{1}(S)=h^{1}(S(-C))+h^{1}\left(S \otimes \mathcal{O}_{C}\right),
$$

if this quantity remained constant in a deformation then the same would be true for each summand, and the first map in the sequence would have to remain surjective.

Proposition 4.9. The Rational Conjecture is true for rational singularities for which $h^{1}(S)=0$ (for example, for the taut singularities classified by Laufer [Lau73]).

Proof. One must show that a non-quasi-homogeneous singularity $(V, 0)$ with $h^{1}\left(S_{X}\right)=0$ must have $h^{1}\left(-\left(K_{X}+E\right)\right)>0$. By Proposition 4.8, it suffices to assume that the graph is not starshaped. Since $H^{1}\left(S_{X}\right) \rightarrow H^{1}\left(\Theta_{E}\right)$ is surjective, $h^{1}\left(S_{X}\right)=0$ implies that all vertices in the graph have valency at most three. So, the graph contains a subgraph

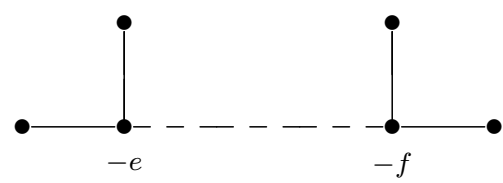

with all negative self-intersections at least two. (Some of these can be taut.) Let $F$ denote the reduced curve connecting the nodes, running from the $(-e)$-curve to the $(-f)$-curve; it suffices to show that $h^{1}\left(-\left(K_{X}+E\right) \otimes \mathcal{O}_{F}\right) \neq 0$. Since $F$ is supported on a cyclic quotient singularity, the sheaf $-\left(K_{X}+E\right) \otimes \mathcal{O}_{F}$ depends only on the numerics of the line bundle $-\left(K_{X}+E\right)$ restricted to $F$. But this line bundle dots to 0 with the interior curves, and to -1 with the nodes; thus, it 


\section{J. WAHL}

equals $\left(K_{X}+F\right) \otimes \mathcal{O}_{F}$, whence

$$
h^{1}\left(-\left(K_{X}+E\right) \otimes \mathcal{O}_{F}\right)=h^{1}\left(\left(K_{X}+F\right) \otimes \mathcal{O}_{F}\right) .
$$

Computing now on a resolution of the cyclic quotient, one has $h^{1}\left(K_{X}\right)=0$, and standard dualities and vanishing give

$$
h^{1}\left(\left(K_{X}+F\right) \otimes \mathcal{O}_{F}\right)=h^{1}\left(K_{X}+F\right)=h_{F}^{1}(\mathcal{O}(-F))=h^{0}\left(\mathcal{O}_{F}\right)=1 .
$$

Example 4.10. Consider a rational singularity with the following graph, where as usual the unmarked bullets are (-2)-curves:

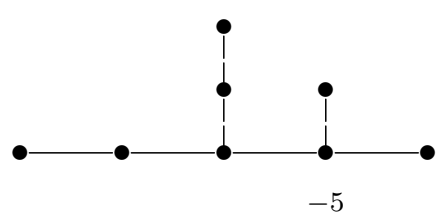

First, we have $h^{1}\left(-\left(K_{X}+E\right)\right)=2$. Starting from the fundamental cycle as in Remark 4.6, one finds $\left[-K_{X}\right] \equiv Y$, which has multiplicity three at the left hand node, one on the four outer vertices, and two on the others. Calculating with $Z \equiv Y-E$, one deduces from Corollary 4.5 that $h^{1}\left(-\left(K_{X}+E\right)\right)=2$. Next, consistently with the conjecture, there is a singularity with this graph and with $h^{1}\left(S_{X}\right)=1$. We can write it as a splice quotient [NW05], that is, the quotient of $x^{3}+y^{3}+z w^{7}=z^{2}+w^{2}+x y=0$ by the discriminant group $G$ (of order 60). Calculating the semi-universal deformation via the computer algebra system Singular, one finds that the only deformation on which $G$ acts equivariantly is obtained by adding $t z^{3} w^{5}$ to the first equation. By general theory, this represents exactly the only equisingular deformation of the original singularity.

Remark 4.11. In a forthcoming paper, we show that the conjecture is true for any graph satisfying $d_{i} \geqslant 2 t_{i}-2$ for all $i$; this is a condition only at the nodes. More precisely, if such a graph is not star-shaped, then $h^{1}\left(S_{X}\right)$ is equal to the number of ends of the graph minus four, which in turn is one less than $h^{1}\left(-\left(K_{X}+E\right)\right)$.

\section{5. $\mathbb{Q}$-Gorenstein smoothings}

Suppose that $(V, 0)$ is an isolated Cohen-Macaulay singularity, whose dualizing sheaf $\omega$ has order $r$. If we write $\left(\omega^{\otimes i}\right)^{* *} \equiv \omega^{[i]}$ and choose an isomorphism $\omega^{[r]} \simeq \mathcal{O}$, the index one cover is the analytic spectrum of $\mathcal{O} \oplus \omega \oplus \omega^{[2]} \oplus \cdots \oplus \omega^{[r-1]}$. The germ $(V, 0)$ is said to be $\mathbb{Q}$-Gorenstein if the index one cover is also Cohen-Macaulay (in which case it is Gorenstein). (Alternatively, $(V, 0)$ is the quotient of a Gorenstein singularity by a finite group acting freely off the singular point.) This is equivalent to requiring that all $\omega^{[i]}$ have maximum depth. In particular, $\omega^{*} \simeq \omega^{[r-1]}$ is Cohen-Macaulay. Recall that a rational surface singularity is $\mathbb{Q}$-Gorenstein.

A $\mathbb{Q}$-Gorenstein smoothing $\pi:(\mathcal{V}, 0) \rightarrow(\mathbb{C}, 0)$ of $(V, 0)$ is one which is the quotient of a smoothing of the index one cover.

Lemma 5.1. Let $\pi:(\mathcal{V}, 0) \rightarrow(\mathbb{C}, 0)$ be a $\mathbb{Q}$-Gorenstein smoothing of the normal surface singularity $(V, 0)$. Then $\alpha_{\pi}=0$.

Proof. As indicated above, the dualizing sheaf $\omega_{\mathcal{V}}^{*} \simeq \omega_{\mathcal{V} / \mathbb{C}}^{*}$ has depth three. Tensoring with $\mathcal{O}_{V}$ 


\section{Milnor AND TJURINA NUMBERS FOR SMOOTHINGS OF SURFACE SINGULARITIES}

gives a depth two subsheaf of $\omega_{V}$ which is equal off the singular point, hence equal. Therefore, $\alpha_{\pi}=0$.

Suppose that $G \subset \operatorname{GL}(n+1, \mathbb{C})$ is a finite group acting freely off the origin, with $n \geqslant 2$. Then $\left(\mathbb{C}^{n+1} / G, 0\right)$ is Cohen-Macaulay, and is Gorenstein if and only if $G \subset \operatorname{SL}(n+1, \mathbb{C})$ (since $G$ contains no pseudo-reflections). Next let $f:\left(\mathbb{C}^{n+1}, 0\right) \rightarrow(\mathbb{C}, 0)$ be a $G$-invariant function having an isolated singularity $(W, 0)$ at the origin, with invariants $\mu$ and $\tau$. The induced map $\bar{f}:\left(\mathbb{C}^{n+1} / G, 0\right) \rightarrow(\mathbb{C}, 0)$ is a $\mathbb{Q}$-Gorenstein smoothing of the quotient singularity $(W / G, 0) \equiv$ $(V, 0)$. The group $G$ acts freely on the Milnor fibre $M$ of $W$, so the new smoothing has Milnor fibre $M / G$, which continues to have (rational) homology only in dimensions zero and $n$. Euler characteristics (and hence Milnor numbers) are related by

$$
1+(-1)^{n} \mu=|G|\left(1+(-1)^{n} \bar{\mu}\right) .
$$

Denote by $\bar{\tau}$ the dimension of the corresponding smoothing component of $V$.

Theorem 5.2. Consider the $\mathbb{Q}$-Gorenstein smoothing above of $(V, 0)$.

(i) If $G \subset \operatorname{SL}(n+1, \mathbb{C})$, then $\bar{\mu} \geqslant \bar{\tau}$, with equality if and only if $(V, 0)$ is quasi-homogeneous.

(ii) If $G \not \subset \mathrm{SL}(n+1, \mathbb{C})$, then $\bar{\mu} \geqslant \bar{\tau}-1$, with equality if and only if $(V, 0)$ is quasi-homogeneous.

We will prove this result via a series of lemmas. Denote by $J$ and $T$ the Jacobian and Tjurina algebras of $f$, respectively. The group $G$ acts on both these spaces.

Lemma 5.3. For the G-invariant subspaces, one has

$$
\operatorname{dim} J^{G} \geqslant \operatorname{dim} T^{G},
$$

with equality if and only if $f$ and hence $V$ are quasi-homogeneous.

Proof. The inequality is clear. The function $f$ is $G$-invariant, so is in $J^{G}$, and is 0 if and only if it is 0 in $J$. If $f$ is quasi-homogeneous, one may choose weights so that $G$ commutes with the $\mathbb{C}^{*}$ action. Thus, $V$ is also quasi-homogeneous.

Let $\tau^{\prime}=\operatorname{dim} T^{G}$, and choose $G$-invariant polynomials $h_{1}, \cdots, h_{\tau^{\prime}}$ inducing a basis of $T^{G}$. The semi-universal deformation of $(W, 0)$ restricts to a family

$$
\mathcal{W}=\left\{f+\sum_{i=1}^{\tau^{\prime}} t_{i} h_{i}=0\right\} \subset \mathbb{C}^{n+1} \times \mathbb{C}^{\tau^{\prime}} \rightarrow \mathbb{C}^{\tau^{\prime}}
$$

on which $G$ acts, trivially on the base and hence on the fibres. This gives a smooth family

$$
(\mathcal{W} / G, 0) \equiv(\mathcal{V}, 0) \rightarrow\left(\mathbb{C}^{\tau^{\prime}}, 0\right)
$$

of deformations of $(V, 0)$.

Lemma 5.4. The family $(\mathcal{V}, 0) \rightarrow\left(\mathbb{C}^{\tau^{\prime}}, 0\right)$ is a full smoothing component of the base space of the semi-universal deformation of $(V, 0)$. In particular, one has $\tau^{\prime}=\bar{\tau}$.

Proof. Denote the relative Kähler differentials of $f:\left(\mathbb{C}^{n+1}, 0\right) \rightarrow(\mathbb{C}, 0)$ by $\Omega_{f}^{1}$, and its dual by $\Theta_{f}$; similarly, one has $\Omega_{\bar{f}}^{1}$ and $\Theta_{\bar{f}}$. By [GL85], the dimension $\bar{\tau}$ is the length of the cokernel of the inclusion

$$
\Theta_{\bar{f}} \otimes \mathcal{O}_{V} \subset \Theta_{V}
$$




\section{J. WAHL}

But $\Theta_{V}=\left(\Theta_{W}\right)^{G}$, since $W$ has an isolated normal singularity, $G$ acts freely off that point, and $\Theta_{W}$ is reflexive and locally free off the singular point. Similarly, $\Theta_{\bar{f}}=\left(\Theta_{f}\right)^{G}$. Thus, $\bar{\tau}$ is the colength of the inclusion

$$
\Theta_{f}^{G} \otimes \mathcal{O}_{W} \subset\left(\Theta_{W}\right)^{G} .
$$

Writing $R=\mathbb{C}\left\{z_{1}, \cdots, z_{n+1}\right\}$ and using the sequence

$$
0 \rightarrow f^{*}\left(\Omega_{\mathbb{C}\{t\}}^{1}\right) \rightarrow \Omega_{R}^{1} \rightarrow \Omega_{f}^{1} \rightarrow 0
$$

and basic homological algebra (for example, [Wah81, $(A .1)]$ with $M=\Omega_{f}^{1}$ and $t=f$ ) gives the exact sequence

$$
0 \rightarrow \Theta_{f} \rightarrow \Theta_{f} \rightarrow \Theta_{W} \rightarrow \operatorname{Ext}_{R}^{1}\left(\Omega_{f}^{1}, R\right) \rightarrow \operatorname{Ext}_{R}^{1}\left(\Omega_{f}^{1}, R\right) \rightarrow T_{W}^{1} \rightarrow \operatorname{Ext}_{R}^{2}\left(\Omega_{f}^{1}, R\right)=0 .
$$

One has a natural $G$-isomorphism $\operatorname{Ext}_{R}^{1}\left(\Omega_{f}^{1}, R\right) \cong J_{f}$, so taking $G$-invariants of the $G$-equivariant exact sequence

$$
0 \rightarrow \Theta_{f} \otimes \mathcal{O}_{W} \rightarrow \Theta_{W} \rightarrow J_{f} \rightarrow J_{f} \rightarrow T_{f} \rightarrow 0
$$

yields

$$
\bar{\tau}=\operatorname{dim} T_{f}^{G}=\tau^{\prime} .
$$

To compare $\mu$ to $\operatorname{dim} J_{f}^{G}$, we relate the action of $G$ on $J_{f}$ with the action on the cohomology of the Milnor fibre (in particular, on $H \equiv H^{n}(M ; \mathbb{C})$.) The $n$-form $d x_{1} \wedge d x_{2} \wedge \cdots \wedge d x_{n+1} / d f$ is holomorphic on the Milnor fibre, and $G$ acts on it via the determinant character. Recall the following result.

Theorem 5.5 (Wall, [Wal80]). The G-modules $H$ and $J_{f} \otimes($ det) are isomorphic, where det is the determinant character.

(This result does not require that $G$ act freely off the origin, only that it leave $f$ invariant.) We conclude as follows.

Lemma 5.6. One has $\operatorname{dim} J^{G}=\operatorname{dim} H^{\nu}$, where $H^{\nu}$ is the isotypic component of $H$ corresponding to the character $\nu=(\operatorname{det})^{-1}$.

The action of $G$ on the full cohomology ring $H^{*}(M)$ can be deduced from the following general result.

Lemma 5.7. Let $X$ be a finite connected $C W$ complex with cohomology $H^{*}(X)$ only in even degrees (respectively, only in odd degrees and degree zero). Suppose that a finite group $G$ acts freely on $X$. Then as a $G$-module, $H^{*}(X)$ is exactly $\chi(X) /|G|$ copies of the regular representation (respectively, the direct sum of two trivial representations and the direct sum of $-\chi(X) /|G|$ copies of the regular representation).

Proof. Let $\phi_{g}: X \rightarrow X$ be the homeomorphism corresponding to $g \in G$. Consider the Lefschetz number

$$
L\left(\phi_{g}\right)=\sum_{i}(-1)^{i} \operatorname{Tr}\left(\phi_{g}^{*}: H^{i}(X)\right) .
$$

The group $G$ acts freely, so by the Lefschetz fixed point theorem, for $g \neq e$ one has

$$
L\left(\phi_{g}\right)=0 \text {. }
$$

Clearly,

$$
L\left(\phi_{e}\right)=L(\mathrm{Id})=\chi(X)
$$




\section{Milnor AND TJuRina NUMBERs FOR SMOOTHINGS OF SURFACE SINGUlARITIES}

When $H^{*}(X)$ is non-zero only in even degrees, one has by definition

$$
L\left(\phi_{g}\right)=\chi(g),
$$

where $\chi$ is the character corresponding to the representation of $G$ on $H^{*}(X)$. Note that the Euler characteristic satisfies $\chi(X)=\operatorname{dim} H^{*}(X)$. Thus, one has $\chi(g)=0$ for $g \neq e$ and $\chi(e)=$ $\operatorname{dim} H^{*}(X)$. Standard character theory implies that the representation is a direct sum of copies of the regular representation.

When $H^{*}(X)$ has cohomology only in odd degrees and degree zero, write $H^{*}(X)=H^{0} \oplus H^{\prime}$, where $H^{\prime}$ is a direct sum of the odd cohomology (which we assume is non-zero). Let $\eta$ be the character corresponding to the representation of $G$ on $H^{\prime}$. Then the argument above yields

$$
\eta(e)=\operatorname{dim} H^{\prime}, \quad \eta(g)=1 \quad \text { if } g \neq e .
$$

This time, character theory implies that the representation of $G$ on $H^{\prime}$ is a direct sum of a trivial representation and $\left(\operatorname{dim} H^{\prime}-1\right) /|G|$ copies of the regular representation. As $\chi(X)=1-\operatorname{dim} H^{\prime}$, the claim easily follows.

Back to the Milnor fibre M, we conclude that

(i) if $n$ is even, then $H^{*}(M)$ is a direct sum of $(1+\mu) /|G|$ copies of the regular representation;

(ii) if $n$ is odd, then $H^{*}(M)$ is a direct sum of two trivial representations and $(\mu-1) /|G|$ copies of the regular representation.

In particular, for $n$ even, every multiplicative character $\chi$ of $G$ occurs in $H^{*}(M)$ with the same multiplicity $(1+\mu) /|G|$, which as mentioned before equals $1+\bar{\mu}$. Thus, the dimension of $H^{\chi}$ is $(1+\mu) /|G|$ unless $\chi$ is the trivial character, in which case the dimension is one less. But the determinant character of $G$ is trivial if and only if $G \subset \operatorname{SL}(n+1, \mathbb{C})$. One now has all the ingredients to verify Theorem 5.2 in this case.

When $n$ is odd, Lemma 5.7 implies that as a $G$-module, $H$ consists of one copy of the trivial representation plus $(\mu-1) /|G|=\bar{\mu}-1$ copies of the regular representation. Thus, one knows the dimension of each isotypic component, and the same argument as in the even case completes the proof of Theorem 5.2.

\section{Normal surfaces in $\mathbb{C}^{4}$}

Let $(V, 0) \subset\left(\mathbb{C}^{4}, 0\right)$ be a germ of a normal surface singularity. If $(V, 0)$ is Gorenstein, it is a complete intersection; otherwise, it is maximal-minor determinantal. The base space of the semiuniversal deformation is smooth, as $T_{(V, 0)}^{2}=0$; computer programs can be used to calculate it. It is unknown whether the Milnor fibre is always simply-connected; this is true for the rational triple points, since one has a unique Milnor fibre, diffeomorphic to the minimal resolution. The Milnor number is difficult to compute. Since $\alpha=0$ in these cases, one wants to prove the following conjecture.

Conjecture 6.1 (Codimension Two Conjecture). Let $(V, 0) \subset\left(\mathbb{C}^{4}, 0\right)$ be a non-Gorenstein singularity. Then

$$
\mu \geqslant \tau-1,
$$

with equality if and only if $(V, 0)$ is quasi-homogeneous.

Example $6.2([\mathrm{Oku} 05,(6.3)])$. There is a $(V, 0) \subset\left(\mathbb{C}^{4}, 0\right)$ which is not $\mathbb{Q}$-Gorenstein and not quasi-homogeneous (though it has a star-shaped graph), with $\mu=\tau$. 


\section{J. WAHL}

Consider the singularity $(V, 0)$ defined by the vanishing of the $2 \times 2$ minors of the matrix

$$
\left(\begin{array}{ccc}
x & y & z \\
y-3 w^{2} & z+w^{3 m} & x^{2}+6 w y-2 w^{3}
\end{array}\right) \text { for } m \geqslant 1 .
$$

The singularity $(V, 0)$ has multiplicity three with $p_{g}=m$, and the same (integral homology sphere) resolution graph as the hypersurface singularity $x^{2}+y^{3}+z^{6 m+7}=0$ (which has $p_{g}=$ $m+1)$ :

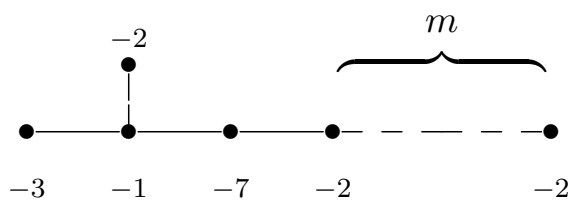

Corollary 6.6 of [Oku05] yields $\mu=12 m+1$. Van Straten has used Singular to prove $\tau=$ $12 m+1$ for $m=1$, and to indicate its likelihood for general $m$. The semi-universal deformation should be obtained by perturbing the matrix by adding

$$
\left(\begin{array}{lll}
0 & 0 & 0 \\
f & g & h
\end{array}\right)
$$

where $f=\sum_{i=0}^{3 m-1} a_{i} w^{i}, g=\sum_{i=0}^{3 m-1} b_{i} w^{i}, h=\sum_{i=0}^{3 m} c_{i} w^{i}+x \sum_{i=0}^{3 m-1} d_{i} w^{i}$. Thus, the $a_{i}, b_{i}, c_{i}, d_{i}$ are parameters for the semi-universal deformation.

\section{ACKNOWLEDGEMENTS}

We thank Shrawan Kumar for the proof of Lemma 5.7 and Duco van Straten and Jacob Fowler for help with computer calculations.

\section{REFERENCES}

BS11 M. Bhupal and A. Stipsicz, Weighted homogeneous singularities and rational homology disk smoothings, Amer. J. Math. 133 (2011), 1259-1297. http://dx.doi.org/10.1353/ajm.2011. 0036

BG80 R.-O. Buchweitz and G.-M. Greuel, The Milnor Number and Deformations of Complex Curve Singularities, Invent. Math. 58 (1980), 241-281. http://dx.doi.org/10.1007/BF01390254

Del73 P. Deligne, Intersections sur les surfaces réguliers, SGA 7 II, 1-38, Springer Lecture Notes 340 (1973).

Gir82 J. Giraud, Improvement of Grauert-Riemenschneider's theorem for a normal surface, Ann. Inst. Fourier, Grenoble 32 (1982), no. 4, 13-23. http://dx.doi.org/10.5802/aif.892

GL85 G.-M. Greuel and E. Looijenga, The dimension of smoothing components, Duke Math. J. 52 (1985), no. 1, 263-272. http://dx.doi.org/10.1215/S0012-7094-85-05214-7

GMP85 G.-M. Greuel, B. Martin, and G. Pfister, Numerische Charakterisierung Quasihomogener Gorenstein-Kurvensingularitäten, Math. Nachr. 124 (1985), 123-131. http://dx.doi.org/10. 1002/mana. 19851240109

Gre80 G.-M. Greuel, Dualität in der lokalen Kohomologie isolierter Singularitäten, Math. Ann. 250 (1980), 157-173. http://dx.doi.org/10.1007/BF02599793

Gre81 On deformation of curves and a formula of Deligne, in Algebraic Geometry (La Rabida, 1981), Springer Lecture Notes 961, 141-168. http://dx.doi.org/10.1007/BFb0071281

GS83 G.-M. Greuel and J.H.M. Steenbrink, On the topology of smoothable singularities, Proc. Symp. Pure Math. 40, Part I (1983), 535-545. http://dx.doi.org/10.1090/pspum/040.1/713090 


\section{Milnor AND TJuRina NUMBERs FOR SMOOTHINGS OF SURFACE SINGUlARITIES}

Hoc77 M. Hochster, The Zariski-Lipman conjecture in the graded case, J. Alg. 47 (1977), 411-424. http://dx.doi.org/10.1016/0021-8693(77)90232-0

Lau73 H. Laufer, Taut two-dimensional singularities, Math. Ann. 205 (1973), 131-164. http://dx. doi.org/10.1007/BF01350842

Loo86 E. Looijenga, Riemann-Roch and smoothings of singularities, Topology 25 (1986), no. 3, 293302. http://dx.doi.org/10.1016/0040-9383(86)90045-5

LS85 E. Looijenga and J. Steenbrink, Milnor number and Tjurina number of complete intersections, Math. Ann. 271 (1985), 121-124. http://dx.doi.org/10.1007/BF01455800

LW86 E. Looijenga and J. Wahl, Quadratic functions and smoothing surface singularities, Topology 25 (1986), 261-291. http://dx.doi.org/10.1016/0040-9383(86)90044-3

NW05 W. Neumann and J. Wahl, Complete intersection singularities of splice type as universal abelian covers, Geom. Topol. 9 (2005), 699-755. http://dx.doi.org/10.2140/gt.2005.9.699

Oku97 T. Okuma, The plurigenera of Gorenstein surface singularities, Manuscripta Math. 94 (1997), 187-194. http://dx.doi.org/10.1007/BF02677846

Oku05 , Numerical Gorenstein elliptic singularities, Math. Z. 249 (2005), no. 1, 31-62. http: //dx.doi.org/10.1007/s00209-004-0688-2

Rei M. Reid, Surface cyclic quotient singularities and Hirzebruch-Jung resolutions, Warwick lecture notes, available at homepages.warwick.ac.uk/ masda/surf/more/cyclic.pdf.

Sai71 K. Saito, Quasihomogene isolierte Singularitäten von Hyperflächen, Invent. Math. 14 (1971), $123-142$.

SSW08 A. Stipsicz, Z. Szabó, and J. Wahl, Rational blowdowns and smoothings of surface singularities, J. of Topology 1 (2008), 477-517. http://dx.doi.org/10.1112/jtopol/jtn009

Vos02 H. Vosegaard, A characterization of quasi-homogeneous complete intersections, J. Algebraic Geom. 11 (2002), no. 3, 581-597. http://dx.doi.org/10.1090/S1056-3911-02-00298-9

Wah75 J. Wahl, Vanishing theorems for resolutions of surface singularities, Invent. Math. 31 (1975), 17-41. http://dx.doi.org/10.1007/BF01389864

Wah76_, Equisingular deformations of normal surface singularities, I, Ann. of Math. 104 (1976), no. 2, 325-356. http://dx.doi.org/10.2307/1971049

Wah80 Elliptic deformations of minimally elliptic singularities, Math. Ann. 253 (1980), 241262. http://dx.doi.org/10.1007/BF0322000

Wah81_, Smoothings of normal surface singularities, Topology 20 (1981), 219-246. http://dx . doi.org/10.1016/0040-9383(81)90001-X

Wah85_, A characterization of quasi-homogeneous Gorenstein surface singularities, Comp. Math. 55 (1985), 269-288.

Wah11_, On rational homology disk smoothings of valency 4 surface singularities, Geom. Top. 15 (2011), 1125-1156. http://dx.doi.org/10.2140/gt.2011.15.1125

Wah13 L Log-terminal smoothings of graded normal surface singularities, Michigan Math J. 62 (2013), 475-489. http://dx.doi.org/10.1307/mmj/1378757884

Wal80 C.T.C. Wall, A note on symmetry of singularities, Bull. London Math. Soc. 12 (1980), 169-175. http://dx.doi.org/10.1112/blms/12.3.169

Wat80 K. Watanabe, On plurigenera of normal isolated singularities, I, Math. Ann. 250 (1980), 65-94. http://dx.doi.org/10.1007/BF02599787

Wat81 K. Watanabe, Some remarks concerning Demazure's construction of normal graded rings, Nagoya Math. J. 83 (1981), 203-211.

Jonathan Wahl jmwahl@email.unc.edu

Department of Mathematics, The University of North Carolina, Chapel Hill, NC 27599-3250, USA 